\title{
Diseño de base de datos para piezas audiovisuales producidas en UNPA- UACO 2004-2016, previo análisis y catalogado
}

\author{
Database design for produced audiovisual pieces At UACO-UNPA, since the 2004-2016 \\ period-UNPA
}

\author{
Lucas Ezequiel Silva Pérez, Marta Bianchi \\ lucas_silva490@hotmail.com, martapilarbianchi@gmail.com
}

Departamento de Ciencias Sociales - Unidad Académica Caleta Olivia - Universidad Nacional de la Patagonia Austral - Acceso Norte, Ruta 3 s/n - Caleta Olivia - Santa Cruz - Argentina

Recibido: 01/07/2019. Aceptado: 07/10/2019

\section{RESUMEN}

Este documento muestra una propuesta para organizar un archivo audiovisual en línea para su consulta, previo trabajo de catalogado y organización de un banco de datos que sistematiza la producción audiovisual constitutiva del patrimonio de la Unidad Académica Caleta Olivia (UACO), Universidad Nacional de la Patagonia Austral (UNPA). El trabajo se ha desplegado como alumno becario en el marco del proyecto de investigación La producción audiovisual en la UNPA- UACO, en el periodo 2004-2016, cuyo objetivo general se vincula con describir las condiciones de producción, prácticas y dinámicas de la realización audiovisual en esa unidad académica.

Uno de los objetivos particulares, sobre el cuál se trabajó concretamente desde esta propuesta, es la creación de un archivo o banco de datos con las producciones del período de referencia, digitalizando cada pieza y organizándolas para conectar con la web facilitando su consulta posterior. Si bien el proyecto de investigación sigue su curso, la discusión generada tuvo incidencia en la forma de catalogado de las producciones y en las decisiones respecto de la manera de organizarlas para que puedan ser consultadas por usuarios en general.

Reviste especial importancia este trabajo, dado que es clave en la preservación de la memoria local, su organización y administración en tanto patrimonio.

Palabras clave: Producciones Audiovisuales; Clasificación; Identidad; Sistematización, Puesta en línea.

\begin{abstract}
This document shows a proposal to organize an online audiovisual archive for consultation, prior cataloging work and organization of a data bank that systematizes audiovisual production constituting the heritage of the Caleta Olivia Academic Unit (UACO), National University of Patagonia (UNPA).The work has been deployed as a student intern in the framework of the research project Audiovisual Production at UNPA-UACO, in the period 2004-2016, whose general objective is to describe the production conditions, practices and dynamics of audiovisual production in that academic unit.

One of the particular objectives, on which we worked specifically from this proposal, is the creation of a file or database with the productions of the reference period, digitizing each piece and organizing them to connect to the web, facilitating their subsequent consultation. Although the research project is ongoing, the generated discussion had an impact on the way
\end{abstract}


catalogs of the productions are made and on the decisions regarding how to organize them so that they can be consulted by users in general.

This work is especially important, given that it is key in the preservation of local memory, its organization and administration as a heritage.

Key words: Audiovisual Productions; Clasification; Identity; Systematization, Online installation.

\section{INTRODUCCIÓN}

El avance de la tecnología y el crecimiento exponencial de material audiovisual ha producido piezas de manera desorganizada, lo que ocasiona problemas para las diversas organizaciones, instituciones u organismos que desean dar cuenta de sus propias creaciones audiovisuales.

La no utilización de herramientas, conocimientos o medios para crear una base de datos donde se organice bajo determinados criterios el material, termina decantando en la utilización de los clásicos medios de difusión a través de la web, tales como YouTube, Vimeo, entre otros.

El caso de la Universidad Nacional de la Patagonia Austral no queda exento de esta problemática: desde el año 2004 hasta la actualidad genera producciones audiovisuales tanto de actividades académicas como de proyectos de extensión y diversas actividades en conjunto con las comunidades locales, que no se encuentran catalogadas ni organizadas en una base que posibilite su consulta y visualización.

Las condiciones de producción de la UNPA UACO desde el 2004 hasta el día de hoy se han ido modificando paulatinamente, permitiendo que el contenido y las producciones audiovisuales se hayan ido transformando en cuanto a calidad y cantidad, a lo largo de los años. Pero también esto genera un problema a la hora de identificar rasgos comunes en las producciones y una forma correcta de catalogación.

Es por ello que la sistematización de las producciones audiovisuales resulta menester a la hora de poder identificarlas, analizar el contexto y condiciones de producción y los rasgos identitarios de la realización audiovisual UNPA UACO.

Mi trabajo como alumno becario se inserta en el proyecto de investigación 29/B211 "La producción audiovisual en la UACO-UNPA, desde el período 2004-2016”.

El objetivo del trabajo se centra en plantear una estrategia para organizar la información en un entorno digital, aplicable a cualquier recurso audiovisual, aplicable a otras entidades de información distintas a la tradicional biblioteca. Se considera a su vez prioritario recuperar las producciones en tanto patrimonio audiovisual cultural para su conservación, sistematización y divulgación, para gestar un Archivo Audiovisual dada la importancia que reviste su utilización tanto presente como futura. Se propone sistematizar las producciones y gestar una interfaz que permita el fácil acceso a la información respecto a las producciones audiovisuales realizadas.

En primer lugar se trabajó en la organización y el catalogado de las 180 piezas audiovisuales que componen el período, tarea liderada por la becaria Viviana Godoy. Luego se discutió y 
propuso el diseño esquemático y funcional de una base de datos la cual pueda ser de acceso público y se encuentre disponible tanto para la comunidad universitaria como para la comunidad en general.

\section{REFERENCIAS}

Las políticas nacionales y los condicionamientos socio económicos han tenido un impacto permanente en el funcionamiento y desarrollo de las Universidades Públicas. Las áreas de Comunicación de las Universidades son permeables a estos cambios, sobre todo en lo referido a la posibilidad de desarrollo de piezas audiovisuales independientes de las contingencias y ritmo que impone el trabajo de difusión de información administrativa y de gestión. Los sectores de comunicación institucional, las carreras de Comunicación Social y/o Audiovisual y otros actores universitarios producen piezas vinculadas con la identidad de la región y con problemáticas sociales del medio. Muchas de esas producciones han sido impulsadas a la luz de la variación tecnológica, legislativa y económica de la última década, especialmente con la creación de los Polos Audiovisuales. Sin embargo, dicha producción no se encuentra en la mayoría de los casos debidamente catalogada y tampoco está completamente dispuesta para su visualización por parte de la comunidad. Es éste el motivo de la actual propuesta: construir una memoria organizada de la producción audiovisual de UNPA -UACO desde 2004.

Se reconoce que el documento audiovisual es todo recurso o soporte en los que se combinan simultáneamente audio y video. Se incluye en ellos, los medios como el cine, la televisión y otros sistemas de carácter didáctico, que utilizan grabaciones y acústicas acompañadas de imágenes. (López Yepes, 2014)

Comprendiendo esto, y en palabras de Edmondson, podemos acercarnos a dar una definición de Archivo Audiovisual:

"Un archivo audiovisual es una organización (o un departamento de organización) cuyo cometido, que podrá estar establecido por ley, consiste en facilitar el acceso a una colección de documentos audiovisuales y del patrimonio audiovisual mediante actividades de acopio, gestión, conservación y promoción.” (Edmondson, Audiovisual Archiving Philosophy Interest Network, \& Unesco, 2008)

Desde el marco de esta investigación se considera que las producciones audiovisuales realizadas en el período de 2004 al 2016 son documentos audiovisuales históricos, que transmiten, conservan y visualizan las actividades sociales, científicas y culturales de la sociedad. A su vez, al enmarcarse dentro de una institución, adquieren verdadero valor de documento social. De allí surge la importancia de la creación de un archivo audiovisual donde se lleve a cabo la conservación, sistematización y facilitación del acceso para su divulgación. Cuando hablamos de sistematización nos referimos específicamente al ordenamiento y clasificación de datos e informaciones, estructurando de manera precisa categorías y relaciones, posibilitando de esta manera la constitución de bases de datos organizadas(Caldera Serrano \& Aranz Escacha, 2013) y (2004) 
Bajo la línea de sistematización y el catalogado de la información, se fue ideando un modo de preservar estos documentos audiovisuales, para ello en primera medida se fueron analizando diversas variantes que afectaban a esta preservación:

- Primero se analizó qué tipo de materiales podíamos encontrar en la vasta colección audiovisual, conociendo el tipo de material existente, se pudo establecer una idea de los soportes que podían darse en este catalogado, tanto físicos como digitales;

- Luego se analizó qué formatos existían en estas piezas audiovisuales, para así poder evaluar y elegir una forma de categorización según género, formato y otras características relevantes.

- Finalmente se ideó un sistema de catalogado para dar forma a un diseño de base de datos donde se almacenaría la información de cada pieza audiovisual con una ficha técnica y su información pertinente en cada una de las mismas.

Este trabajo permitió la realización de una base de datos tangible en donde se encuentran todas y cada una de las producciones audiovisuales realizadas en la UNPA UACO, en el período de 2004 al 2016 y que proporcionó el basamento para que se pensara la creación de una interfaz web en donde alojar dichas producciones bajo el régimen del catalogado y sistematización aplicada.

Hasta la fecha se ha llegado a un catalogado de alrededor de más de 180 piezas audiovisuales, entre las que surgen varios formatos y temáticas. De esto mismo hace un análisis y desarrollo más exhaustivo Godoy Viviana en su Informe de Plan de Trabajo denominado "Análisis y catalogado de piezas audiovisuales para diseño de base de datos producción audiovisual UNPA-UACO 2004-2016", con el cual colaboré.

Con fundamento en la propuesta mencionada, se utilizaron un conjunto de formatos y géneros definidos para catalogar todas las producciones audiovisuales de la UNPA-UACO.

Se propone partir de tres principales categorías que son transversales a estas piezas audiovisuales. Hablamos así de los "Formatos" de tipo informativo-periodístico, series y documental. Estos tres formatos comprenden todo el Archivo Audiovisual de la institución y de los mismos se desprenden las demás categorías, comprendidas en "Temas": cultural, social, recreativo, artístico, institucional, político, científico.

Esta base de datos, junto con el catalogado propuesto se pretende alojarlo a través de la página web de la Unidad Académica, que posibilite al usuario visualizar el contenido de la misma.

Dentro de lo correspondiente a las facultades que tendrá el usuario dentro de esta interfaz web, se encuentra la posibilidad de visualizar cada una de las producciones audiovisuales de la UNPA-UACO; se podrá utilizar un gestor de búsqueda de acuerdo a formatos y temáticas antes mencionadas o a través de palabras claves (keywords) que el usuario ingrese para facilitar la búsqueda de un determinado Archivo Audiovisual; se podrá obtener de cada Archivo Audiovisual una descripción de la información pertinente a cada pieza audiovisual; pre-visualizar los últimos archivos añadidos a la interfaz web; poder compartir las producciones audiovisuales a través de las distintas redes sociales.

Contar con la posibilidad de la creación de una interfaz web constituye un registro sólido de la memoria institucional, el cual le brinda un importante valor simbólico a la misma y a su vez permite que se identifiquen a lo largo del tiempo rasgos comunes que van caracterizando dichas producciones audiovisuales a lo largo del tiempo. 
A su vez, se puede destacar la importancia que reviste la creación de esta interfaz gráfica para los alumnos de la Licenciatura en Comunicación Audiovisual. Los mismos pueden contar con un espacio digital en el que alojar sus producciones y visualizarlas a través de la web. Contar con un Archivo Audiovisual de producciones de los alumnos es de suma importancia ya que en ellas también se evidencian estos rasgos identitarios y aportan a la definición y construcción de una identidad desde lo audiovisual en la institución y en la región.

La importancia de la creación de una interfaz web radica en la posibilidad de crear un acceso tangible de los usuarios a través de internet a la información y la cultura. El acceso es también una parte del proceso de preservación. Dar acceso al material audiovisual es el principal objetivo de la preservación, ya que el acceso es la forma de compartir este contenido con el público al que se encuentra dirigido.

$\mathrm{Al}$ respecto, Ray Edmondson plantea:

El acceso es cualquier forma de uso de la colección de un archivo, servicios, conocimientos, incluyendo la reproducción a tiempo real del sonido y la imagen en movimiento, y referencias a fuentes de información sobre esos contenidos de sonidos $e$ imagen, y las áreas temáticas que representan.

Las formas de acceso en los archivos, pueden ser iniciadas o propiciadas por la institución, como serían las proyecciones, o por el usuario, haciendo una demanda de material. Por ello es que la UNPA UACO toma la iniciativa en crear un espacio propicio para el acceso a las piezas audiovisuales, pensando a su vez en el aprovechamiento de las tecnologías existentes y el alcance que puede permitir la utilización de una plataforma web.

El Archivo de la UNPA-UACO contaría con su propia plataforma digital, en la cual se sube contenido a la misma a través de otra plataforma de videos (http://youtube.com), como ya lo viene trabajando desde hace un tiempo hacia acá, y son categorizados según un criterio determinado. Cada video cuenta con una descripción y ficha de contenidos y las etiquetas a los cuales se encuentra asociado el mismo. Otras características del sitio sería la posibilidad de ver el último contenido agregado y además poder compartirlo a través de diversas redes sociales.

De esta plataforma digital se proyecta la visualización gráfica que podría llegar a asumir el Archivo Audiovisual para difundir y sistematizar el material de la institución, a través de un fácil acceso. La posibilidad de elegir un archivo, ya sea desde el género, temática o algún tipo de etiqueta, permitiría al usuario una fácil búsqueda del material. La posibilidad de contar con una breve descripción y datos técnicos específicos, permitirían la posibilidad de tener un primer acceso rápido y sencillo a la información que se desee buscar. Trabajar a la par con la plataforma Vimeo, permite una rápida subida del material disponible para su visualización en la plataforma. Este documento explica la propuesta aunque ésta no ha podido concretarse dado que al momento de la escritura no se cerraron los acuerdos formales con el adminstrador de la página web de la institución. 
 \\ UNIDAD ACADEMICA CALETA OLIVIA}

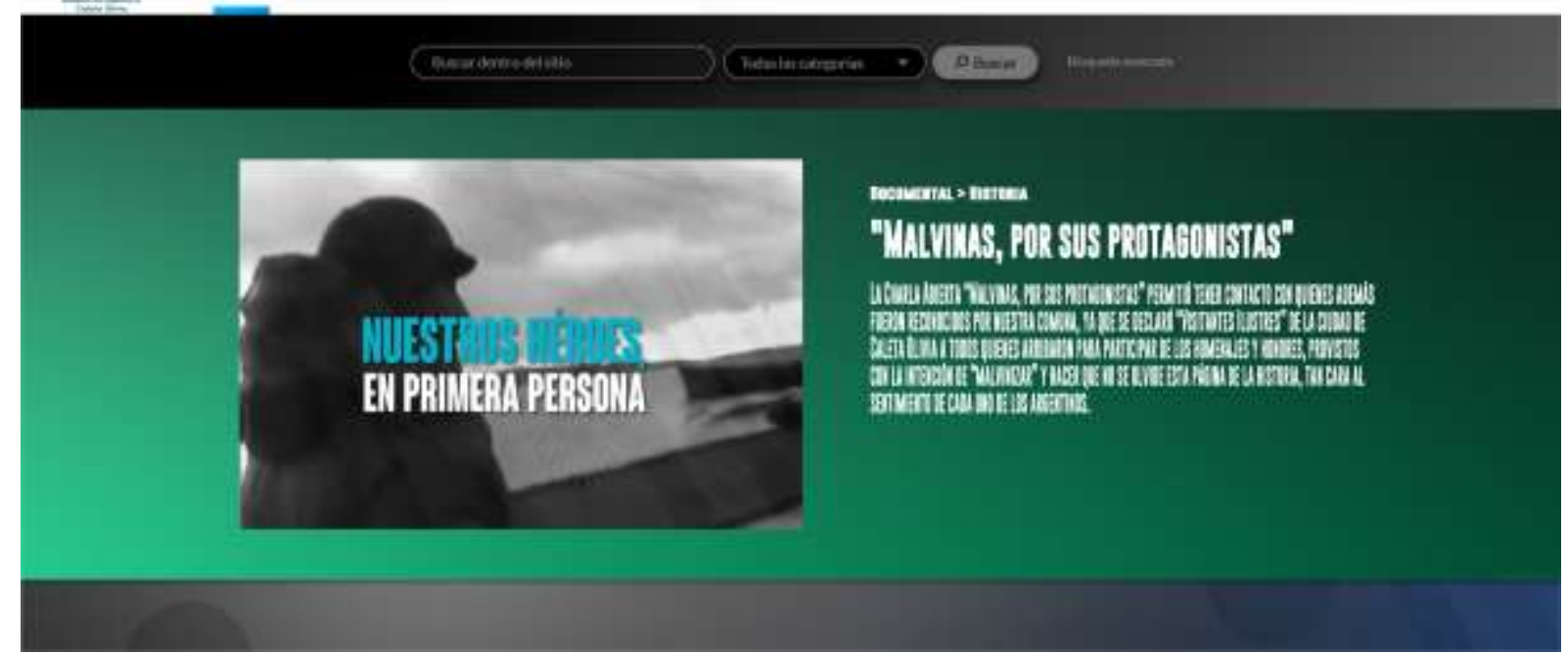

Propuesta de página de inicio

ARCHIVO AUDIOVISUAL.

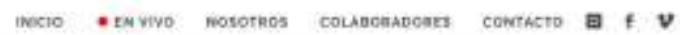

\section{>UITIMOS COKTEMIDOS ARADIDOS}

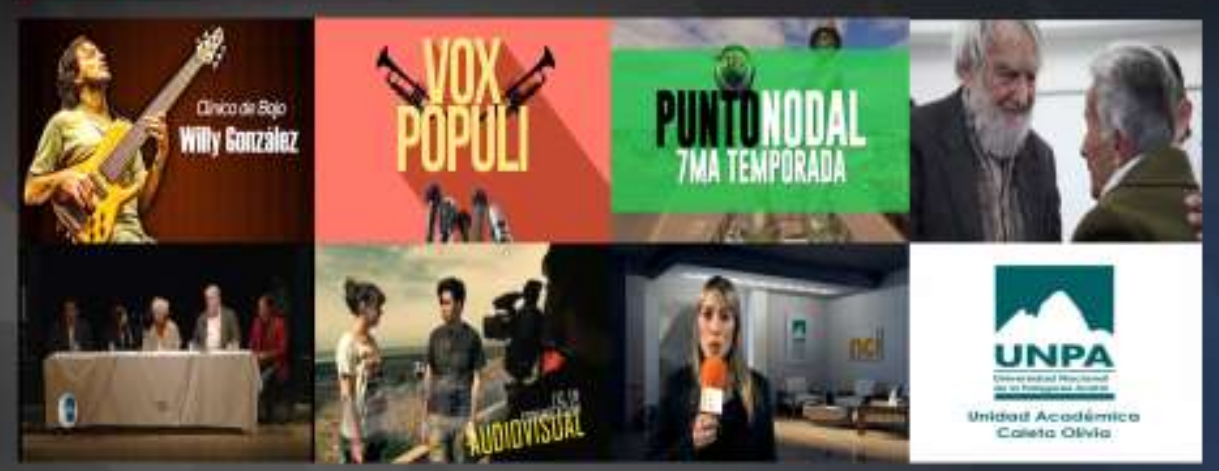

Propuesta de interfaz con botones según series producidas y catalogadas 


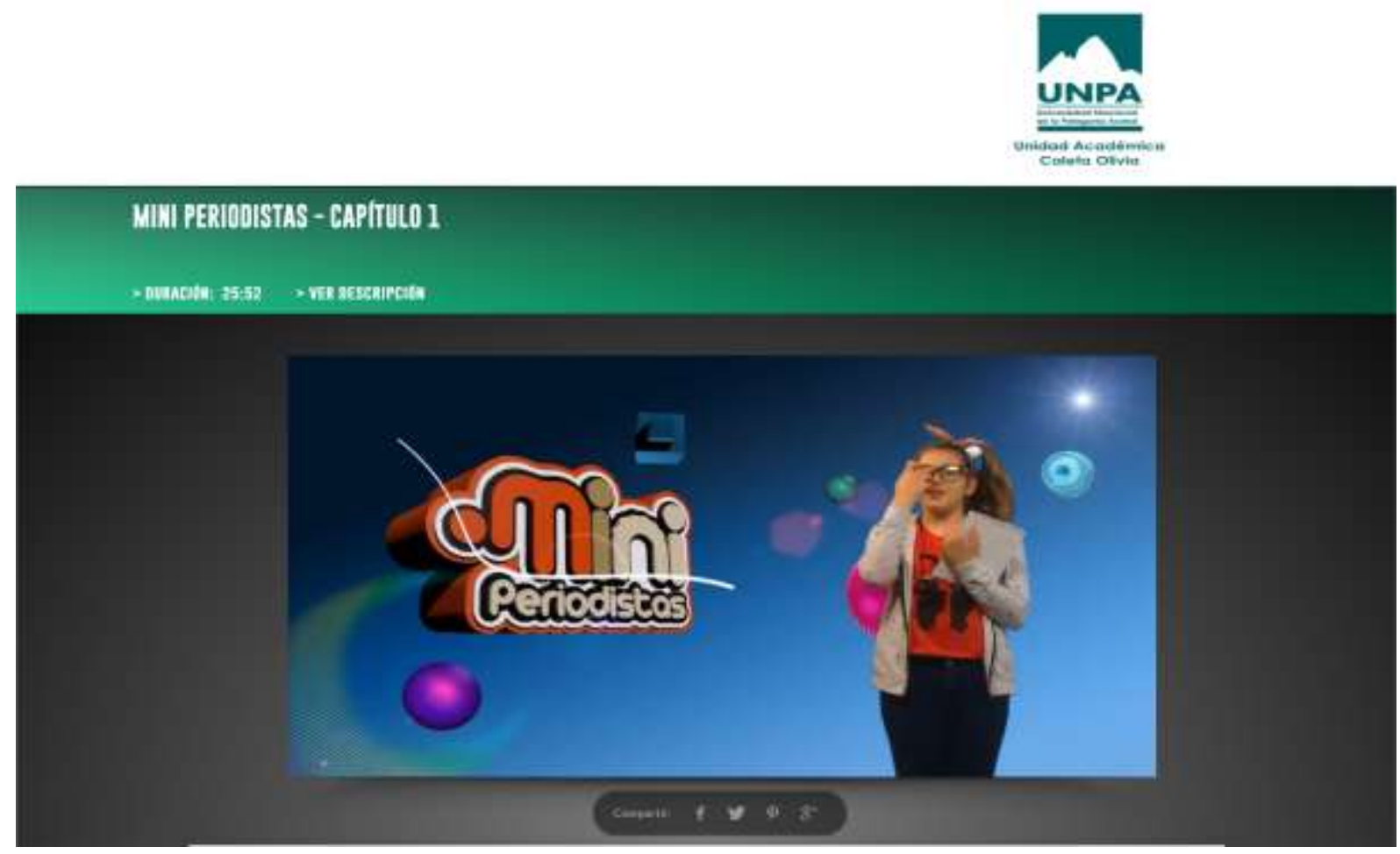

Propuesta de visualización de cada pieza

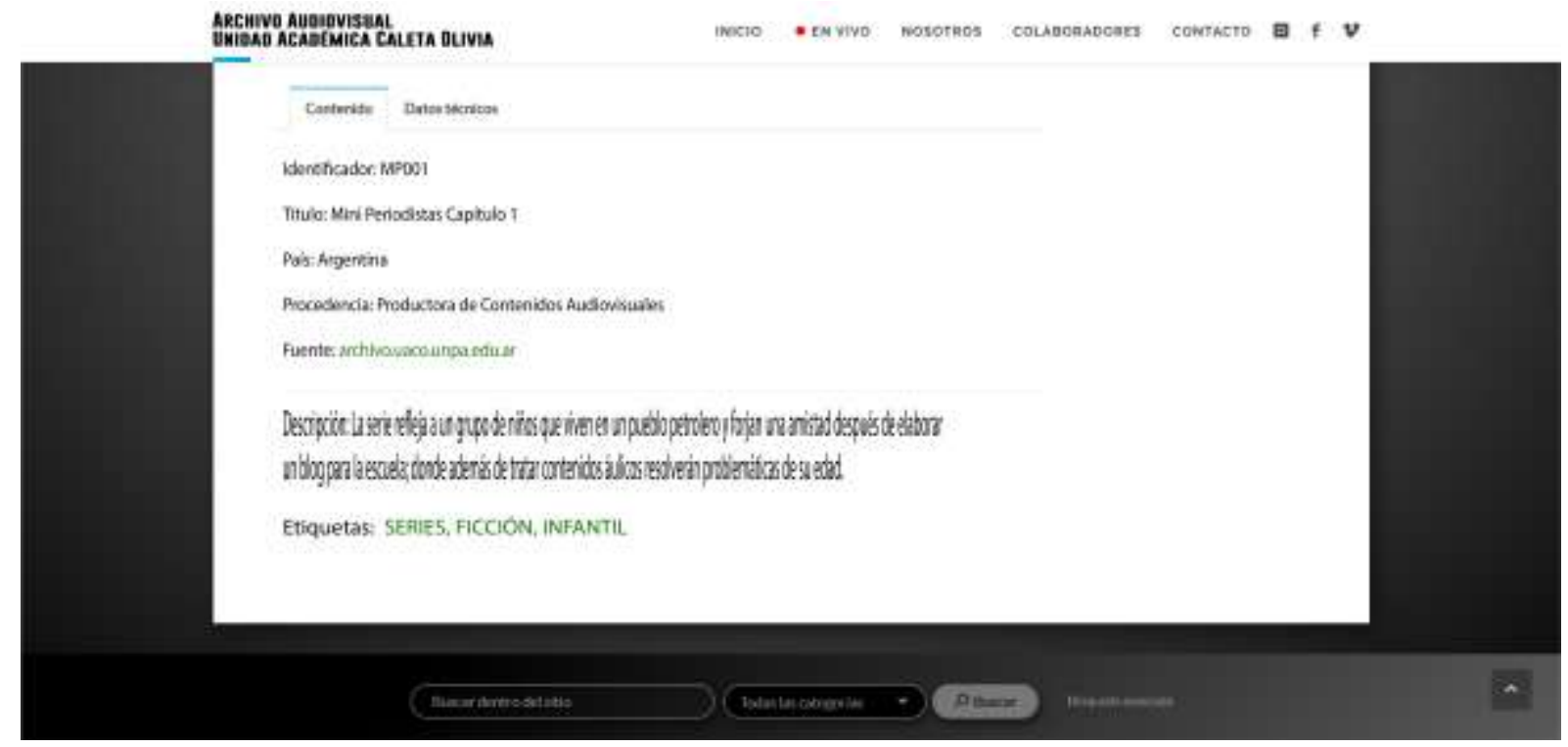

Propuesta de visualización de ficha audiovisual 


\section{CONCLUSIONES}

El resultado de este trabajo es un aporte consistente a la memoria colectiva regional en general y a la memoria institucional en particular. La importancia del patrimonio audiovisual como parte de esa memoria es un dato que exige a la institución su catalogado para ponerlo a disposición de la comunidad toda.

Hablar de patrimonio es hablar necesariamente de investigación, de inventario, de objetos materiales e inmateriales, de monumentos, de obras de arte, de documentos, de música, de tradiciones y del paisaje que los contiene. Al margen de su naturaleza, el término patrimonio encierra en su significado un sentido de bien heredado, de transmisión generacional que le confiere un valor añadido.(Salvador Benitez, A., 2017)

El patrimonio audiovisual cobra sentido pleno cuando es reconocido, valorado y apropiado por la comunidad de la cual forma parte. La convergencia tecnológica ha posibilitado que los productos audiovisuales puedan estar al alcance de un click y por tanto que su apropiación sea colectiva, a decir de Casacuberta ((2003)) "las obras culturales de la cultura digital ya no se construyen en forma individual, solipsista, sino de forma colectiva, organizada". Nuestro trabajo es de alguna manera el de poner a disposición este patrimonio que contribuye al desarrollo integral de nuestra sociedad, en el contexto de estos nuevos recursos tecnologicos.

Se ha logrado organizar el $80 \%$ de la producción audiovisual generada en la Unidad Académica Caleta Olivia entre los años 2004 y 2016. Se ha organizado a partir de ello un archivo por género/temática que posibilita el armado de una propuesta de consulta en línea. En paralelo, se han llevado adelante entrevistas con referentes institucionales que reflexionan sobre las condiciones político-económicas en las que se gestaron los productos, la estructura y equipamiento, capacitación y consolidación de grupos que permiten generar los productos audiovisuales de UNPA y de la Unidad Académica Caleta Olivia específicamente. Por otra parte, en el seno del trabajo del equipo se han sistematizado las cuestiones relativas a las variaciones del contexto legislativo y condiciones histórico-institucionales, que condicionaron la producción audiovisual. Esta reflexión, que enmarca el trabajo de catalogado y archivo, aspira a ser una contribución a las experiencias que en la región trabajan en la misma línea.

La clasificación a la que se ha llegado es amplia y variada. Considerando esto, podemos decir que las producciones audiovisuales en la institución han ido mutando a lo largo del tiempo, intentando abarcar diversos ámbitos y esferas de lo social, de acuerdo al contexto bajo el cual se encontraban enmarcados y a su vez, de acuerdo a las condiciones de producción que fueron variando. Podemos agregar que, tanto el contexto socioeconómico como las condiciones de producción reinantes, van a ir contruyendo una imagen de la institución, representada a través de sus producciones audiovisuales.

Este proyecto nos permitió crear una mirada crítica sobre los rasgos que asumen las producciones audiovisuales de la Universidad, como así también poder brindar la posibilidad de que estas piezas audiovisuales se encuentren a disposición de un mayor número de personas y dicho conocimiento pueda ser compartido.

Así entonces, podemos decir que este proyecto permitió visibilizar las producciones audiovisuales realizadas por la UNPA UACO, como las mismas se fueron dinamizando a 
través del tiempo y a su vez facilitar la creación de un entorno por medio de una base de datos que permita el acceso al patrimonio audiovisual de esta institución.

El poder trabajar en conjunto con la Universidad, permitió que las producciones audiovisuales lleguen a un mayor abanico de grupos sociales, sobretodo académico; pensar en el desarrollo de una plataforma web personal para alojar el Archivo Audiovisual permitiría un mayor manejo del Archivo Audiovisual para la institución, aunque demandaría iniciar un nuevo proyecto desde cero.

Las nuevas producciones audiovisuales se deberían realizar teniendo ya en cuenta el sistema de catalogado pensado durante el proyecto, para que a la hora de cargar información en la base de datos ya se cuente con un método sistematizado.

\section{REFERENCIAS}

CALDERA SERRANO, J., \& Aranz Escacha, P. (2013). Documentación audiovisual en televisión. Recuperado de http://www.digitaliapublishing.com/a/21090/

CALDERA SERRANO, J., \& Nuño Moral, M. V. (2004). Diseño de una base de datos de imágenes para televisión. Gijón (Asturias): Ediciones Trea.

CASACUBERTA SEVILLA, D. (2003). Creación colectiva: En Internet el creador es el público. Barcelona: Gedisa Editorial.

EDMONDSON, R., Audiovisual Archiving Philosophy Interest Network, \& Unesco. (2008). Filosofía y principios de los archivos audiovisuales. México, D.F.: Fonoteca Nacional.

LÓPEZ YEPES, J. (2014). Diccionario enciclopédico de ciencias de la documentación. Madrid: Síntesis. https://doi.org/10.22201/iibi.0187358xp.2005.38.4064

SALVADOR BENITEZ, A. (2017). Investigación y Docencia sobre Patrimonio Audiovisual. VII Jornadas de celebración del Dia del Patrimonio Audiovisual, 6-15. Madrid: Biblioteca Nacional de España. https://doi.org/10.25145/j.pasos.2019.17.016 